\title{
4th Symposium on \\ Primary Breast Cancer in Older Women
}

\section{Theme: Putting personalising care into practice}

East Midlands Conference Centre, Nottingham, UK

3rd March 2017

Under the auspices of:

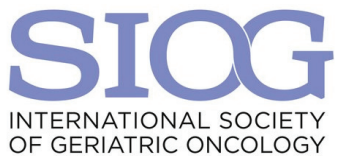

Accredited by the Royal College of Surgeons of England for up to 5 CPD points

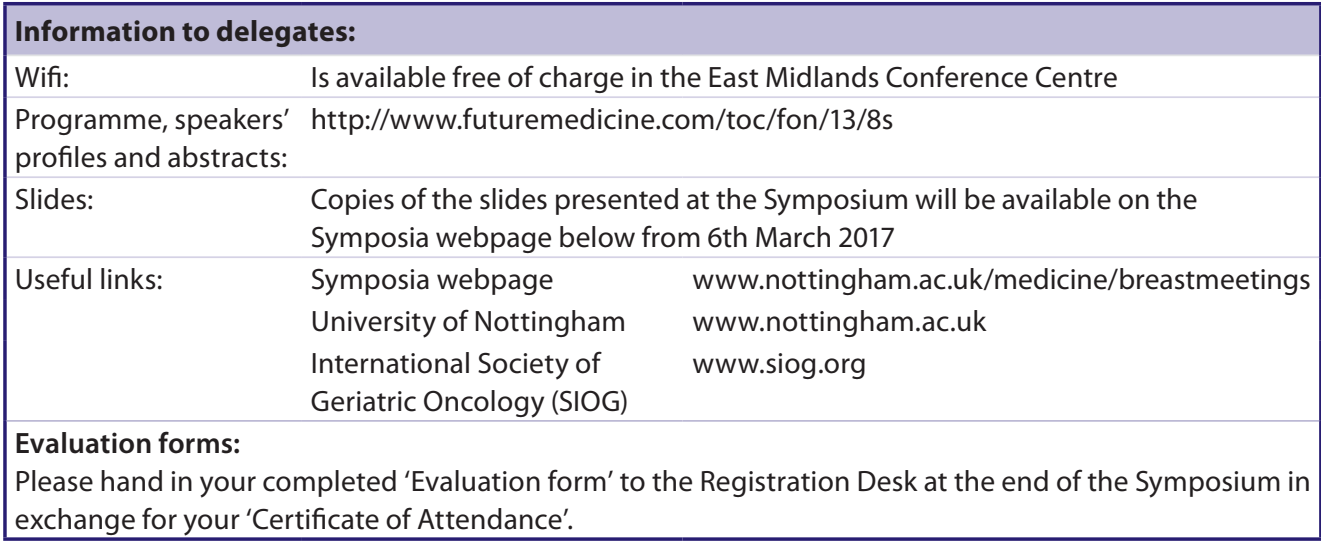

Future $\because$ Medicine part of 
Welcome message

We would like to offer a very warm welcome to the "4th Symposium on Primary Breast Cancer in Older Women'.

As you know this is the fourth meeting of this kind, following its inception in 2010 [1]. Since 2013, the event has been hosted by the University of Nottingham under the auspices of the International Society of Geriatric Oncology (SIOG), and in 2015, we introduced a geriatrician into the faculty.

Over these years, we have generated sufficient interest in this area and now is the time to start thinking of how to put what we have learned from experts into our daily clinical practice, which we believe should focus on the individual needs of the patients. As a result, we have given this year's Symposium the theme, 'Putting personalising care into practice'. Also, for the first time, we have included both oral and poster presentations from submitted abstracts, which were reviewed and selected by the faculty and the editorial team of Future Oncology. This allows more sharing of research findings and good practice from the delegates. We continue to have a multidisciplinary faculty, which, on this occasion, also includes a plastic surgeon and a geriatric oncology nurse for the first time. They are introduced in the 'speakers' profile'. We are also pleased to announce that we have invited Miss Ruth Parks, a core surgical trainee, to join the organising committee.

Finally, we are indebted to our sponsors and would encourage you to visit their stands between sessions. We hope that you will enjoy, not only the day, but also the interactions with one another, including our patients during the meeting.

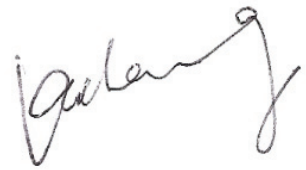

Mr KL Cheung

Co-chair

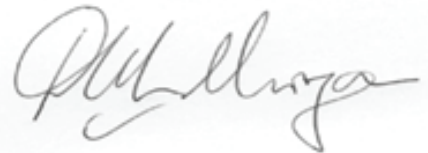

Dr DAL Morgan

Co-chair

\section{Reference}

1 Cheung KL, Ellis IO, Morgan DAL et al. Optimising the management of primary breast cancer in older women - a report of a multi-disciplinary study day. Breast 20, 581-584 (2011). 


\title{
4th Symposium on Primary Breast Cancer in Older Women
}

\author{
THEME: $\quad$ Putting personalising care into practice \\ HOST: $\quad$ University of Nottingham, UK \\ CO-CHAIRS: KL Cheung and DAL Morgan \\ DATE: $\quad$ Friday 3rd March 2017 \\ VENUE: $\quad$ East Midlands Conference Centre (EMCC), Nottingham, UK
}

\section{Objectives:}

With an overall aim to personalise the management of primary breast cancer in older women, this event has the following specific objectives:

- To develop an in-depth understanding around the biology and treatment options;

- To explore the specific physical and psychosocial needs and consideration including patients perspective;

- To gain insight into the development of a holistic and multidisciplinary management approach and the importance of supporting research.

\section{Target participants:}

- Members of multidisciplinary clinical and research teams with an interest in optimising the management of primary breast cancer in older women - breast surgeons, oncologists, geriatricians, pathologists and clinical nurse specialists, as well as patient representatives;

- We welcome both UK and international delegates.

\section{Formats:}

As before, the day features presentations (in the form of lectures from external and local faculty) with a number of interactive discussions and sharing of patients' experience. For the first time both oral and poster presentations are included, with abstracts published as a Supplement to Future Oncology (impact factor 2.129 [2015]) and citable in PubMed/MEDLINE).

\section{Auspices and CPD accreditation:}

SIOG auspices have been approved. The meeting is accredited by the Royal Colleges of Surgeons of England (which implies reciprocal recognition by other medical royal colleges) for up to $5 \mathrm{CPD}$ points.

\section{Organising committee:}

Kwok-Leung Cheung (co-chair)

David Morgan (co-chair)

Ruth Parks

Lesley Randall 
Programme:

Arrival and registration

Welcome and introduction (09:45-10:00)

\begin{tabular}{|llll|}
\hline & Presentation & Speaker & Affiliations \\
\hline 09:45-10:00 & Welcome and introduction & Kwok-Leung Cheung & Clinical Associate Professor, \\
& & University of Nottingham \\
& & Consultant Breast Surgeon, \\
& & Royal Derby Hospital \\
& & UK National Representative, SIOG \\
& & Symposium co-chair \\
\hline
\end{tabular}

\section{Session 1: Local and systemic therapies (10:00-11:15); Chair: David Morgan}

\begin{tabular}{|llll|}
\hline & Presentation & Speaker & Affiliations \\
\hline 10:00-10:25 & $\begin{array}{l}\text { Selecting for chemotherapy } \\
\text { - the use of genomics }\end{array}$ & Etienne Brain & $\begin{array}{l}\text { Medical Oncologist, } \\
\text { Institut Curie, Paris, France } \\
\text { Immediate Past President, SIOG }\end{array}$ \\
& Selecting for radiotherapy & Philip Poortmans & $\begin{array}{l}\text { Professor of Radiation Oncology, } \\
\text { Radboud University, } \\
\text { The Netherlands }\end{array}$ \\
& & $\begin{array}{l}\text { President, European Society for } \\
\text { Radiotherapy and Oncology } \\
\text { (ESTRO) }\end{array}$ \\
\hline 10:50-11:00 & Discussion & & \\
\hline
\end{tabular}

Coffee break and poster viewing (11:00-11:30)

Session 2: 'New' ideas (11:30-12:45); Chair: Ruth Parks

\begin{tabular}{|c|c|c|c|}
\hline & Presentation & Speaker & Affiliations \\
\hline 11:30-11:55 & $\begin{array}{l}\text { Geriatrician in the } \\
\text { preoperative assessment } \\
\text { clinic }\end{array}$ & Beatriz Korc-Grodzicki & $\begin{array}{l}\text { Chief, Geriatrics Service, Memorial } \\
\text { Sloan Kettering Cancer Center, } \\
\text { New York, USA }\end{array}$ \\
\hline 11:55-12:05 & Discussion & Panel & \\
\hline \multirow[t]{5}{*}{ 12:05-12:45 } & Oral abstracts & & \\
\hline & $\begin{array}{l}\text { A joint geriatric oncology } \\
\text { clinic for the management } \\
\text { of elderly women } \\
\text { diagnosed with breast } \\
\text { cancer: the Brighton } \\
\text { experience }\end{array}$ & Fiammetta Ugolini & $\begin{array}{l}\text { Brighton and Sussex University } \\
\text { Hospital, UK }\end{array}$ \\
\hline & $\begin{array}{l}\text { The management of breast } \\
\text { cancer in elderly patients: } \\
\text { a local audit }\end{array}$ & Taner Shakir & Peterborough Breast Unit, UK \\
\hline & $\begin{array}{l}\text { Elders with breast cancer } \\
\text { tend to delay seeking } \\
\text { medical care and present } \\
\text { with a later stage }\end{array}$ & Janice Tsang & $\begin{array}{l}\text { Li Ka Shing Faculty of Medicine, } \\
\text { The University of Hong Kong, } \\
\text { Hong Kong SAR }\end{array}$ \\
\hline & $\begin{array}{l}\text { Potential impact of } \\
\text { treatment on the mood of } \\
\text { older women of primary } \\
\text { breast cancer }\end{array}$ & Ruth Parks & $\begin{array}{l}\text { School of Medicine, University of } \\
\text { Nottingham, UK }\end{array}$ \\
\hline
\end{tabular}

Lunch break and poster viewing (12:45-13:45) 
Session 3: Patients and carers (13:45-15:10); Chair: Etienne Brain

\begin{tabular}{|llll|}
\hline & Presentation & Speaker & Affiliations \\
\hline 13:45-14:35 & $\begin{array}{l}\text { Interviewing patients and } \\
\text { carers }\end{array}$ & Heather Stone & $\begin{array}{l}\text { Lead Breast Care Nurse Specialist, } \\
\text { Royal Derby Hospital }\end{array}$ \\
& $\begin{array}{l}\text { The role of the geriatric } \\
\text { oncology nurse }\end{array}$ & Cindy Kenis & $\begin{array}{l}\text { Geriatric Oncology Nurse, } \\
\text { University Hospitals Leuven, } \\
\text { Belgium }\end{array}$ \\
\hline 15:00-15:10 & Discussion & Panel & \\
\hline
\end{tabular}

Coffee break and poster viewing (15:15-15:40)

Session 4: Challenging areas (1540-1645); Chair: Kwok-Leung Cheung

\begin{tabular}{|c|c|c|c|}
\hline & Presentation & Speaker & Affiliations \\
\hline 15:40-16:05 & $\begin{array}{l}\text { Selecting for breast } \\
\text { reconstruction }\end{array}$ & Graeme Perks & $\begin{array}{l}\text { Consultant Plastic and } \\
\text { Reconstructive Surgeon, } \\
\text { Nottingham University Hospitals } \\
\text { Immediate Past President, British } \\
\text { Plastic Reconstructive and } \\
\text { Aesthetic Surgeons (BAPRAS) }\end{array}$ \\
\hline $16: 05-16: 30$ & $\begin{array}{l}\text { Treating HER2 positive } \\
\text { disease }\end{array}$ & Etienne Brain & \\
\hline $16: 30-16: 40$ & Discussion & All faculty & \\
\hline $16: 40-16: 45$ & $\begin{array}{l}\text { Conclusion - summary and } \\
\text { ways forward }\end{array}$ & David Morgan & $\begin{array}{l}\text { Consultant Clinical Oncologist } \\
\text { (Retired), Nottingham University } \\
\text { Hospitals } \\
\text { Symposium co-chair }\end{array}$ \\
\hline
\end{tabular}

\section{Close}


The '4th Symposium on Primary Breast Cancer in Older Women' has been supported by the following companies, to whom we are grateful:

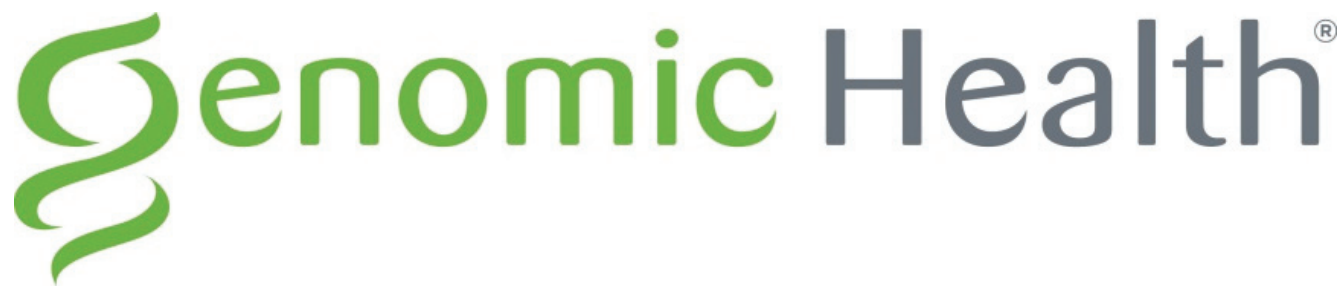

Ariane

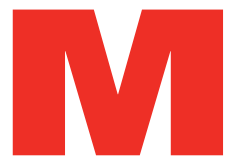

MERMAID

$M E D \mid C A L^{\oplus}$

CROSSING EXPERTISE

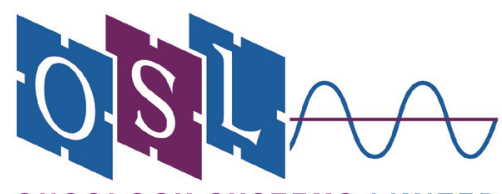

ONCOLOGY SYSTEMS LIMITED
AstraZeneca What science can do

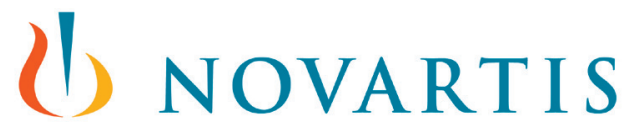

\section{Roche}




\section{SPEAKERS' PROFILES}
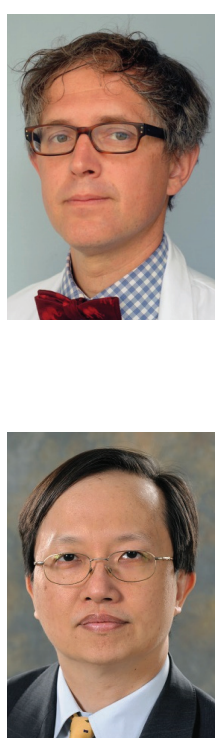

\section{Kwok-Leung Cheung}

Kwok-Leung Cheung, qualified and trained in Hong Kong, was appointed as Consultant Breast Surgeon, Nottingham University Hospitals in 2001. He joined the University of Nottingham in 2004. In 2011, he became a member of the Derby Breast Unit. In 2007 he was selected as an International Guest Scholar of the American College of Surgeons. He is part of the UK National Cancer Peer Review team, and also a member of a number of national and international societies, including the International Society of Geriatric Oncology where he is currently the UK National Representative and a member of the Surgical Task Force.

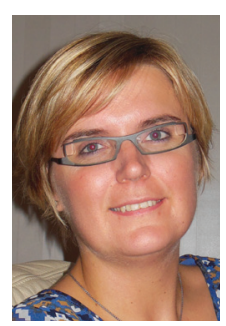

\section{Cindy Kenis}

Cindy Kenis (PhD, MsN, RN) works as a geriatric oncology nurse at the University Hospitals Leuven, Belgium. She coordinated large multicentric studies (2009-2011: 10 Belgian hospitals; 2012-2015: 22 Belgian hospitals) focusing on the implementation of a systematic geriatric screening and assessment within the Belgian cancer population. Her daily clinical practice and research is fully related to this topic. During the past years, she has given several national and international lectures and presentations on to this topic. She's also frequently asked to give educational sessions in different kinds of settings for medical students, nursing students, and other professionals.

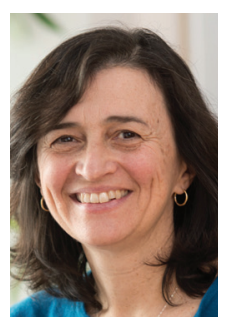

\section{Beatriz Korc-Grodzicki}

Beatriz Korc-Grodzicki is the Service Chief of the Geriatrics at the Memorial Sloan-Kettering Cancer Center and Professor of Clinical Medicine at Weil Cornell Medical College in New York (USA). As an internist with a subspecialty in Geriatrics, she has expertise in treating complex cases with multiple health conditions and provides comprehensive guidance that can help prevent avoidable complications. Over the last few years, she has been dedicated to the care of older adults with cancer, has been a panel member of the NCCN Senior Adult Oncology Guidelines, belonged to the Cancer and Aging Interest Group at the American Geriatric Society and the Geriatric Oncology Special Interest Group at ASCO. She both spearheads clinical research and collaborates with oncologists nationwide in the hunt for best practices in caring for older cancer patients.

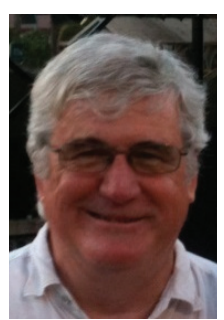

\section{David AL Morgan}

David AL Morgan is a Radiation Oncologist and co-founder of Nottingham's multidisciplinary clinic for the management of older patients with primary breast cancer. He has a career-long interest in breast cancer and belief in the need to empower patients in treatment decision-making, has been active in numerous national and international committees, has been a contributor to many major clinical trials, and has collaborated closely with the University of Nottingham's Translational Radiation Biology Research Group. He has an extensive list of publications in these various areas, as well as other fields of Oncology. 


\section{SUPPLEMENT}

\section{SPEAKERS' PROFILES (CONT.)}

\section{Ruth Parks}

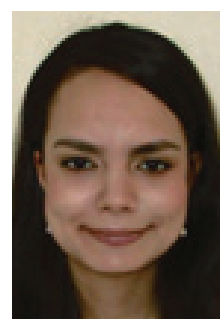

Graeme Perks

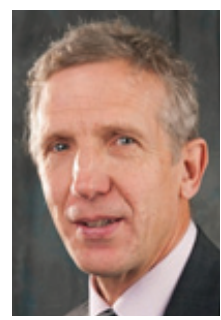

Graeme Perks is Head of the Department of Plastic and Reconstructive Surgery at Nottingham University Hospital's NHS Trust (UK). He is past President of BAPRAS, the national body for plastic surgery, and a member of the Board of ICOPLAST the International Confederations of Plastic Surgery Societies. Graeme helped to develop the Breast Reconstruction service in Nottingham into a centre of repute (along with Mr KL Cheung and Dr David Morgan), but has now retired from full-time practice to make way for his former trainees who now surpass him. He continues to work with the breast reconstruction service in Mansfield.

\section{Philip Poortmans}

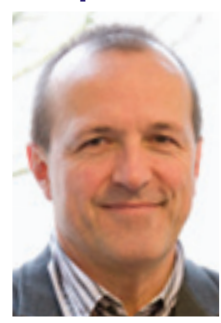

Philip Poortmans is a radiation oncologist whose special interests include breast cancer, quality assurance in clinical trials, malignant lymphoma and rare tumours. He is Executive and Head of the Radiation Oncology Department of the Radboud university medical center in Nijmegen (The Netherlands) - moving soon to Institut Curie in Paris (France). Philip is current chair of the Oncopolicy Committee of ECCO and was recently elected as ECCO President, serving in 2018 and 2019. He is the past President of the European Society for Radiotherapy and Oncology (ESTRO) and active in the ESTRO School of Radiotherapy and Oncology. He is also involved in the European Organisation for Research and Treatment of Cancer (EORTC) as a member of the Radiation Oncology, Lymphoma and Breast Groups, and until recently of the Quality Assurance Committee.

\section{Heather Stone}

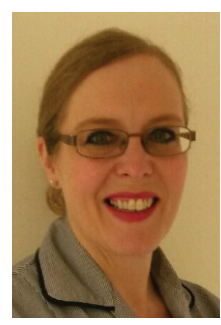

Heather Stone qualified as a Registered Nurse in September 1987 following 3 years' training at Harefield, Hillingdon and Mount Vernon Hospitals. She has predominately worked in the surgical setting taking up Staff Nurse posts on a mixed surgical ward at Mount Vernon Hospital, Harefield ITU and a cardio-thoracic ward at Kings College Hospital, London. In 1991, she trained as a midwife at Kings College Hospital, London, qualifying in 1992. Following a move to Derby in that same year she returned to nursing, firstly as a District Nurse then hospital-based at the Derby City Hospital now re-named The Royal Derby Hospital. She joined the breast unit at the Royal Derby Hospital in Sept 2002 as a breast care nurse, where she has remained in post, being promoted to Lead Specialist Nurse in January 2013. 


\section{LECTURE ABSTRACTS}

\section{L1. Selecting for chemotherapy - the use of genomics}

\section{Etienne Brain}

The development of genomic tools for the elderly has unfortunately not been more successful than what has been observed for new therapies during the last two decades. Although breast cancer is the most developed model, most programs have barred patients older than 65-70 years of age from enrollment in key trials, repeating the unbalance of existing prognostic and predictive algorithms based predominantly on relatively young patients, and so prone to error when applied/extrapolated to the elderly. Adjuvant! Online provides a good example with overestimation of survival.

However, these signatures have been spotlighted to improve the use of healthcare resources, carrying considerable hope and expectation. Since the molecular biology of tumours per se is not very different in the elderly compared with younger ones, genome expression profiling would offer a great opportunity for more accurate prediction and prognosis also in older patients, on the proviso that new genome-based algorithms would factor in/give appropriate weighting to age and comorbidities, lower life expectancy and competing causes of mortality.

So far, two large series focusing on the use of OncotypeDx for elderly breast cancer patients have been presented at conferences. One large (2000 women) French trial using genomic grade index (GGI) as prognosticator to investigate the added value of adjuvant chemotherapy in luminal cases has just completed accrual (ASTER 70s). These will be discussed during the meeting to illustrate the unmet needs and remaining uncertainties.

\section{L2. Selecting for radiation therapy}

\section{Philip Poortmans}

Treatment for low-risk breast cancer patients is a matter of debate. Several trials evaluated the contribution of radiation therapy (RT) after local excision and adjuvant endocrine treatment, showing a limited absolute benefit in terms of local control and no benefit in overall survival.

In interpreting these results, we should mind the generalisability for the whole elderly low-risk population: firstly because generally a disproportionately high very low risk population was recruited; secondly because of the uncertain benefit from endocrine therapy for these patients, even more in view of the competing causes of death. Indeed, the elderly low-risk patients form a unique population regarding prognosis and potential comorbidities, indicating that minimising treatment to safeguard and maintain quality of life without compromising survival is crucial.

With improvement in RT techniques, side effects are reduced and, simultaneously, the burden of RT could be lessened by using shorter (hypofractionated) treatment schedules and by the introduction of accelerated partial breast irradiation for selected patients with early-stage BC.

As on the one hand nor endocrine treatment, nor RT has a demonstrated clinically relevant survival benefit in low-risk patients, and on the other hand withholding both treatments together is associated with a high local recurrence risk, the burden and side effects of both treatments should be used to select one of both treatment options, taking into account comorbidities, life expectancy and personal preferences for care. The final decision should follow a shared-decision making process involving the patient and the multidisciplinary oncology team. Many patients might opt to avoid the possible and mostly long-lasting side effects from prolonged endocrine therapy and prefer a short course of RT, while other patients might go for adjuvant systemic therapy, in which case the added value of RT might be limited.

Finally, treatment decisions should be based on biological rather than formal age, and 'fit' elderly patients should receive identical treatments as their younger counterparts. 


\section{L3. Geriatrician in the preoperative assessment clinic}

\section{Beatriz Korc-Grodzicki}

The aging of the population is a real concern for surgical oncologists, who are increasingly being asked to treat patients who would not have been considered for surgery in the past. In many cases, decisions are made with relatively little evidence, most of which was derived from trials where older age was a limiting factor for recruitment. Effective surgery requires safe performance as well as reasonable postoperative life expectancy and maintenance of quality of life. Treatment decisions for vulnerable elderly patients should take into account data obtained from the evaluation of geriatric syndromes, such as frailty, functional and cognitive limitations, malnutrition, comorbidities and polypharmacy, as well as social support. Postoperative care should include prevention and treatment of complications seen more frequently in the elderly, including postoperative delirium, functional decline and the need for institutionalisation.

Frailty assessment is essential. Poor understanding of frailty is, to a large degree, responsible for undertreatment. For example, too often primary endocrine treatment is preferred to surgical resection for elderly women with breast cancer. On the other hand, there can be overtreatment when frailty is not identified and patients are treated aggressively, resulting in increased mortality, substandard quality of life, and high monetary costs.

Surgery remains the best modality for treatment of solid tumours, and chronologic age alone should not be a determinant for treatment decisions. With adequate perioperative risk stratification, functional assessment, and oncologic prognostication, elderly cancer patients can do as well in terms of morbidity and mortality as their younger counterparts. If surgery is determined to be the appropriate treatment modality, patients should not be denied this option because of their age.

\section{L4. The role of the geriatric oncology nurse}

\section{Cindy Kenis}

The aging of the population results in an increasing number and proportion of older patients diagnosed with cancer. We are challenged to explore new approaches for the care of this particular patient population. Instead of focusing on the medical part and the cancer diagnosis, older persons need a more holistic approach that focuses on a combination of medical, social, functional, cognitive, mental and nutritional needs. Performing a 'comprehensive geriatric assessment' (CGA) to get this holistic view on the patient is therefore recommended.

There are several reasons for treating physicians to integrate the implementation of CGA in their care for older patients with cancer:

I. CGA in older patients with cancer reveals previously unknown geriatric problems in a large proportion of patients can alter cancer treatment decisions, and as a consequence tackling these detected geriatric problems may lead to a better quality of care;

II. CGA is important for further actions. The most important step is the use of the knowledge gathered from the detected geriatric problems by treating physicians. Geriatric recommendations need to be set op and targeted interventions need to be established;

III. Several studies show that CGA can have predictive capacity for toxicity, functional decline or decrease in quality of life (QoL);

IV. CGA has important prognostic information: limitations in most aspects of GA (e.g., cognition, nutrition, functional status, comorbidity) have been shown to be important predictive factors for diminished overall survival (OS).

There are several ways of implementing GA in geriatric oncology: the geriatric oncology unit, geriatric consultation team (GCT) and comprehensive cancer centers where geriatric expertise is not nearby. All models have advantages and disadvantages, and preference should be given to models that fit with the local health care structure and setting which are often very region/country specific (e.g., North America, Canada, Australia, France, Belgium). A nurse, ideally with both geriatric and oncology expertise, plays in key role in all existing models. 


\section{L5. Selecting for breast reconstruction}

Graeme Perks

Breast reconstruction has transformed many patients' lives for the better but (sadly) a small number for the worse. There are many reasons for the rise in the number of breast reconstruction operations over the last 30 years (my lifetime in Plastic Surgery), but credit should go to greater public awareness as the most important. Breast reconstruction is available for both breast conservation and mastectomy procedures, but you need an expert to help put the options into perspective because we all recognise that, "If all you have is a hammer, everything looks like a nail." In helping each woman deliberate about the merits of reconstruction, my interest is in their lifestyle first and the tumour second. This is always in collaboration with the oncologist, both surgical and nonsurgical. The patient activities, habitus, comorbidities, and breast volume will influence discussion of appropriate technique. The contralateral breast is a key consideration because of the difficulties that asymmetry causes in clothes. Timing the reconstruction around adjuvant treatment continues to challenge even the best-informed patient, and debate in relation to the timing of radiation therapy in particular. Increasing obesity and diminishing funding present two challenges for the future.

\section{L6. Treating HER2-positive disease}

Etienne Brain

Although older women are more likely to have breast tumours expressing hormonal receptors, $10-15 \%$ of them present with a HER2 + phenotype. Pivotal trials have inconsistently included few patients aged over 65 years, always highly selected, so that real-life data provide essential complementary information. Assessing the left ventricular function (preferentially through ultrasound) is an essential prerequisite because of the cardiovascular risk increasing with age, with the use of anti-HER2 therapy, and depending also on potential prior exposure to chemotherapy. The International Society of Geriatric Oncology (SIOG) support the use of anti-HER2 treatment in general, both in the adjuvant and metastatic settings for older patients as in younger adults, but only for fit individuals. This is especially true for dual blockade (pertuzumab + trastuzumab) with taxane or for T-DM1 in the metastatic setting. For vulnerable or frail patients, the tricky choice for the right chemotherapy to partner with antibodies makes attractive combinations with less aggressive cytotoxic agent as vinorelbine or capecitabine, or with endocrine therapy in case of expression of hormonal receptors. Trastuzumab alone should be reserved for frail elderly patients at high risk of side effects. Lapatinib has a higher risk of side effects and drug interactions in elderly because of frequent polypharmacy and diarrhea, which may induce dehydration. Although docetaxel and cyclophosphamide plus trastuzumab given for 1 year appears as the most validated regimen for fit early-stage breast cancer in older patients, an alternate regimen such as weekly paclitaxel plus trastuzumab might prove similarly efficient. Adjuvant trastuzumab for less than 1 year remains a research question, especially for patients who are not fit or at cardiac risk, those with small and/or node negative tumours, or those in countries where there are cost issues. The landmark challenge remains whether progress in targeting the HER2 pathway through multiple blockades will soon allow omission of chemotherapy. 


\title{
SUPPLEMENT
}

\section{ORAL ABSTRACTS}

\section{A joint geriatric oncology clinic for the management of elderly women diagnosed with breast cancer: the Brighton experience}

\author{
F Ugolini', J Wright ${ }^{1} \&$ M Reed ${ }^{1}$ \\ 'Brighton and Sussex University Hospital, UK
}

Background: Breast cancer incidence in older individuals is increasingly common in clinical practice, with $30 \%$ of new diagnosis occurring in women aged over 70 years. The 2012 multidisciplinary SIOG and EUSOMA task force recommends considering primary endocrine therapy in those older women with ER-positive tumours with an estimated life expectancy $<2-3$ years, who are unfit for, or who refuse, surgery. Life expectancy is difficult to estimate and it should not be confused with chronological age. Functional and cognitive status may be a more useful indicator of suitable therapeutic options. Formal geriatric assessment in a joint clinic setting may enhance the overall management of these patients and help treatment selection for breast cancer. For these reasons a dedicated clinic has been established in Brighton in which selected patients are assessed jointly by a geriatrician and a breast surgeon. Methods: This cohort study aims to examine the impact of a geriatric-oncology consultation on patients' breast cancer and overall management. Referral criteria: 70 years or older; newly diagnosed with breast cancer, considered unfit for or declining surgery; patients on primary endocrine treatment who develop disease progression. Results: 74 new referrals were seen from June 2015 to October 2016 (age range; 70-98 years). 16 patients (21.6\%) subsequently underwent surgical treatment. Seven patients underwent wide local excision and nine had mastectomy (one bilateral). Regarding axillary treatment, nine patients underwent sentinel node biopsy, three had clearance and four had no axillary procedure. Of those not treated surgically four (9\%) had their endocrine treatment changed, two (3\%) were referred for intravenous biphosphonate and three (4\%) for palliative radiotherapy. 34 patients (46\%) had their medical care modified. Discussion: Assessment in the joint clinic resulted in more patients receiving surgical treatment. Our rate of conversion to surgery fits with data reported in literature.

\section{O2. The management of breast cancer in elderly patients: a local audit}

\section{T Shakir ${ }^{1} \&$ S Goh}

'Peterborough Breast Unit, UK

Background: The management of breast cancer in the elderly varies greatly. We evaluated our practice in this retrospective audit. Methods: All cancers diagnosed in patients aged 75 or over between January 2011 and December 2015 were included. Clinic letters, histology reports and medical notes were analysed for data collection. A self-determined weighting was added to the number of comorbidities if a patient was frail, care resident, using mobility aid or smoker. Results: 366 patients were identified, four were male. $93 \%$ of patients presented through the symptomatic service. The mean age of diagnosis was 83 years (75-102 years). The average number of comorbidities was 2.7 (0-10). Median follow-up was 32 months (0-70 months). The mean lesion size was 29 mm (6-160 $\mathrm{mm})$. There were $70 \%$ invasive ductal carcinoma, 10\% lobular carcinoma, 5\% DCIS, 5\% mucinous, $1 \%$ papillary and $8 \%$ were of a mixed type. $27 \%$ were grade 3 tumours. $85 \%$ of tumours were oestrogen receptor positive and $7 \%$ were HER 2 receptor positive. $40 \%$ of the study population had proven nodal metastases. 201 (55\%) patients underwent surgery as first-line treatment. Four of these patients had breast reconstruction. The overall complication rate was $11 \%$. 23\% of patients who were deemed fit declined surgery. Three patients suffered local recurrence following curative surgery. 157 (43\%) patients received primary endocrine therapy. Radiotherapy was given to 133 (36\%) patients, and only 21 (6\%) patients received chemotherapy. 78 (21\%) patients have passed away. Discussion: There were more comorbidities in primary endocrine group (3.1 vs 2.4 surgery). Mortality rate was higher in primary endocrine group (28\% vs $17 \%$ surgery). The rate of surgical complications was similar in all patients up to 90 years old. However, the complication rate increases in those over the age of 90 years. Designated pathways and joint care between surgeons and specialist physicians may encourage the uptake of surgery. 


\section{O3. Elders with breast cancer tend to delay seeking medical care and present with a later stage}

Janice Tsang ${ }^{1}$, Polly Cheung ${ }^{2}$, Hang-mei Lee², Gary Tse², Sam Choy², Lorna Wong ${ }^{2}$, Maria Shiu², Thomas Yau \& Chun-chung Yau 'Li Ka Shing Faculty of Medicine, The University of Hong Kong, Hong Kong SAR

${ }^{2}$ Hong Kong Breast Cancer Foundation, Hong Kong SAR

Background: Breast cancer risk increases with age and it is anticipated that the number of elderly affected by breast cancer will increase with time. The aims of this study include (1) assessing the tumour characteristics and treatment practice, and (2) investigating the impact of comorbidity in local elderly breast cancer patients. Methods: 13,265 patients with breast cancer diagnosed between 2006 and 2015 from the Hong Kong Breast Cancer Registry were studied. Among them, 861 patients were aged 70 years and above. Results: Upon first symptoms onset, more elderly patients delayed for more than a year in seeking medical consultation (17.7 vs $10.8 \%$ for patients of all ages; $\mathrm{p}=0.005)$; these elderly patients tended to be diagnosed with stage III or IV disease, compared with the elderly patients who sought medical consultation within 3 months (29.7 vs $14.5 \%$; $=0.068$ ). Compared with patients of all ages, invasive tumours in elderly patients exhibited more favourable biological features, including more grade 1 tumours (26.5 vs $19.2 \%$; $\mathrm{p}<0.001)$ and absence of lymphovascular invasion (75.6 vs 68.1\%; $\mathrm{p}<0.001)$. More invasive tumours in elderly patients were positive for oestrogen receptor ( 83.0 vs $78.1 \%$; $\mathrm{p}=0.001$ ), progesterone receptor (70.8 vs $66.0 \%$; $\mathrm{p}=0.006$ ) but HER2 negative (83.6\% vs. $78.7 \% ; \mathrm{p}=0.001)$ compared with patients of all ages. Elderly patients received more mastectomies ( 81.7 vs $57.3 \%$ for patients of all ages; $\mathrm{p}<0.001$ ) but less chemotherapy and radiotherapy. Furthermore, elderly patients with more comorbidities received more conservative treatment. Discussion: Our results demonstrated that elderly patients tended to delay in seeking medical consultation, yet they received less aggressive cancer modalities compared with patients of all ages. Comorbidity may have association with the treatment choices among elderly patients. Although age is an important factor to consider in decision-making for cancer treatment, it must not be the sole guiding principle.

\section{Potential impact of treatment on the mood of older women of primary breast cancer}

\section{RM Parks ${ }^{1}, \mathrm{~K} \mathrm{Cox}^{2}, \mathrm{P} \mathrm{Howard}^{1} \& \mathrm{KL}$ Cheung $^{1}$}

'School of Medicine, University of Nottingham, UK

${ }^{2}$ School of Health Sciences, University of Nottingham, UK

Background: Treatment decision-making in older women with primary breast cancer poses different challenges when compared to their younger counterparts. There is a growing body of evidence for use of comprehensive geriatric assessment (CGA) to help identify potential surgical candidates. Methods: A prospective, two-centre pilot study was conducted evaluating a previously validated, cancer-specific CGA tool in older women with primary (stage I/II) breast cancer. CGA was conducted within 6 weeks and again at 6 months postdiagnosis. The decision of primary treatment followed consultation with the clinical team and was not guided by CGA. Total CGA scores included assessment of: activities of daily living, independent activities of daily living, performance status, comorbidity, mood, social activity and support. Results: 60 patients who had completed CGA at 6 weeks and 6 months postdiagnosis were available for analysis. 42 of these underwent surgery and 18 nonsurgery (primary endocrine therapy). Average age was 80 years (range: $68-92$ years). There was a significant $(\mathrm{p}=0.009)$ improvement in total CGA score between the two time points for nonsurgery patients; total CGA score of 191/263 (6 weeks) compared with 209/263 (6 months). This was not apparent for the surgical patients. Analysis of the individual components of the CGA (listed above) for nonsurgery patients showed a significant $(\mathrm{p}=0.045)$ improvement in mood score from 77/102 (6 weeks) to 85/102 (6 months). No significant changes in other CGA components were noted. Discussion: In this small pilot study, a short-term improvement in mood has been observed in patients undergoing nonsurgery. This may be related to the effects of treatments (endocrine therapy and/or a 'lack of' surgery). The study is ongoing and is expanding into a multicentre one. 


\section{SUPPLEMENT}

\section{POSTER ABSTRACTS}

\section{P1. Bridging the Age Gap in Breast Cancer (BTAG) trial update}

Karen Anna Collins', Maria Burton', Kate Joanna Lifford ${ }^{2}$, Malcolm Walter Ronald Reed ${ }^{4}$, Fiona Armitage ${ }^{3} \&$ Lynda Wyld $^{2}$; on behalf of the

\section{BTAG Team}

'Centre for Health and Social Care Research, Sheffield Hallam University, Collegiate Crescent, Sheffield, UK

2Division of Population Medicine, School of Medicine, Cardiff University, Cardiff, UK

${ }^{3}$ Academic Unit of Surgical Oncology, University of Sheffield, Medical School, Beech Hill, Sheffield, UK

${ }^{4}$ University of Sussex, Brighton and Sussex Medical School, Falmer, Brighton, BN1 9PX, UK

Introduction: One third of all breast cancers occur in women over 70 years of age. Surgery with adjuvant antioestrogens and primary endocrine therapy have equivalent overall survival rates; however, local control rates are superior in surgically treated patients. For women at intermediate risk of surgery there are a complex series of trade-offs to be made in deciding between the two treatments. The decision about whether or not to have chemotherapy is also complex as the risks are higher and the benefits smaller among this age group. Currently, there is little evidence to support clinicians/patients with these treatment decisions. This trial aims to evaluate the effectiveness of decision support interventions (DESIs) to assist in the treatment decision-making of older women with early breast cancer. The DESIs include two patient decision aids (primary endocrine therapy vs surgery plus adjuvant endocrine therapy and chemotherapy vs no chemotherapy) and a clinical treatment outcomes algorithm for clinicians. Methods \& analysis: This multicentre, parallel group, pragmatic cluster randomised controlled trial (RCT) has recruited $46 \mathrm{UK}$ breast sites cluster randomised to either usual practice for women ( $\leq 70$ years) with breast cancer (control) or, usual practice plus the use of the DESIs (intervention). The primary outcome being QoL. Secondary outcomes include decision quality/regret, anxiety, illness perceptions and coping. A process evaluation is being undertaken to assess the implementation, usefulness and acceptability of the DESIs and examine the facilitators/barriers to embedding the DESIs into everyday clinical practice. Results: The trial opened in January 2016 and closes in December 2017. We aim to recruit 1500 women in total (750 per group). Based on our current recruitment data this will be achievable. Discussion: This trial will determine the effectiveness of the implementation of two DESIs on healthcare outcomes and will provide high quality evidence to support treatment decision making among this group of women.

\section{P2. Treatment decisions: factors that influence older women with primary breast cancer \\ Penny Howard', Karen Cox² \& Kwok-Leung Cheung' \\ 'School of Medicine, University of Nottingham, UK \\ ${ }^{2} \mathrm{School}$ of Health Science, University of Nottingham, UK}

Background: Primary endocrine therapy (PET) offers a viable alternative to surgery for older women diagnosed with oestrogen receptor-positive primary breast cancer. It has been established that treatment decisions are influenced by healthcare professional (HCP) preference and patient choice. The factors that influence the HCP treatment preferences have been explored; however, it is unknown what factors influence and facilitate older women's breast cancer treatment decisions. Methods: This study prospectively explored older women's experience of diagnosis and what factors influenced their treatment decisions, to increase understanding and inform decision-making interventions. A longitudinal, qualitative approach was taken. Older women with primary breast cancer (stage I/II) offered either PET or surgical treatment were interviewed at the time of diagnosis and 6 months after treatment. Results: 31 women (aged 70-92 years) were interviewed. Grounded theory was used to analyse data. Emerging themes were:

- Breast cancer is not a big deal: compared to life events and comorbidities. Although most did not feel ready to die, there was an acceptance of mortality, recognising that treatment decisions would have been different earlier in life.

- Appraising the evidence: Older women considered their ability to cope with treatment and resources available. As well as relying on HCP to provide information, women drew upon other's cancer experiences, providing a frame of reference.

- Balancing the risks/benefits of treatment: Weighing up the risk of mortality from cancer against the desire to preserve physical and social functioning, women selected only treatment considered absolutely 'necessary'. Unnecessary risks that threatened independence and potentially burdened others were avoided, allowing normal living to resume swiftly.

Discussion: Older women consider multiple physical and psychosocial factors and wish to be involved in treatment decisions. Reflecting on their experience, women did not regret their treatment choice. There is opportunity to develop decision-making interventions to assist older women to appraise the treatment options and consider the risks of breast cancer treatment to make an individualistic choice. 
P3. Being elderly shouldn't mean inferior breast cancer follow up: our experience of setting up a nurse led one stop clinic for elderly patients with oestrogen receptor positive breast cancer treated with primary hormonal manipulation

Rachel King $(\mathrm{BCN})^{1} \&$ Rachel Bright-Thomas ${ }^{1}$

'Worcestershire Breast Unit, UK

Background: Worcestershire treats over 600 new breast cancers a year. With a greater percentage of our population being over 70 than the UK average (13.4\% in 2011) and with a projected proportional increase of $43 \%$ in the $>85$-year age group by 2021 , we see many elderly ladies with breast cancer who decline surgery for a variety of reasons. We have a register of $>50$ such women maintained on primary hormonal manipulation (PHM) alone since 2014. After surgery for breast cancer, follow-up is relatively easy and infrequent, being once a year with clinical assessment and mammogram for 5 years. However, in women on PHM it is more complex. They may require more frequent follow-up to assess response and adjust treatment. They may also need ultrasound assessment rather than mammography to avoid unnecessary irradiation. We felt that these women and their carers were making more visits than absolutely necessary to a busy clinic with limited time available for discussion and scanning. Methods: We decided to set up and audit the outcome of a one-stop nurse led clinic. We developed new literature specifically for elderly women choosing between PMH or 'surgery plus adjuvant treatment'. We assessed patient's understanding of the information. We arranged nurse-led follow-up in small clinics with plenty of time to allow for mobility and hearing issues. Results: Scans and follow-up were done on the same morning by the same people each time. Patients were called a few days prior to their appointments to ensure there were no health, transport or social issues that would prevent them attending. Any treatment failures were discussed in MDT and dealt with in the most appropriate clinic. Discussion: Patients and the local support group have found this an excellent service and we would like to commend it to other units.

\section{P4. Retrospective audit of treatment for breast cancer in elderly patients at George Eliot Hospital, Nuneaton}

T Sein', H Croft ${ }^{1}$, R Nangalia' \& K Makam

'Breast Unit, Nuneaton, UK

Background: People with early invasive breast cancer, irrespective of age, are offered surgery, radiotherapy and appropriate systemic therapy, unless significant comorbidity precludes it (NICE). The aim of this audit is to evaluate the treatments offered to older women at our unit and compare with the national figures. Methods: Retrospective data collected from 1/10/13-30/9/14: Patient demographics, comorbidities and type of operative procedure. Results: $64 \%$ of patients over 70 years of age and $99 \%$ of those under 70 years of age had surgical treatment, which is comparable to the UK average. $97 \%$ of Patients between 70 and 79 years of age had surgery, which is higher than UK average of 74\%. More breast conservation was seen in older age group (1:3.86 vs 1:2.27), which is significantly higher than the UK average (1.13). Discussion: Patient comorbidities, advanced stage of the cancer and patient wishes are identified as reasons for adopting nonoperative treatment.

P5. Bone health in older breast cancer patients undergoing standard surgical treatment: an audit of guideline and protocol compliance

C Bowler ${ }^{1}$, M D'Auria', Lisa Sawers' $^{1}$, Susie Laking ${ }^{1} \&$ L Whisker $^{1}$

'The Nottingham Breast Institute, Nottingham University Hospitals NHS Trust, UK

Background: Aromatase inhibitors (AI) are recommended for adjuvant treatment of postmenopausal patients with oestrogen receptor-positive breast cancer. However, these drugs are known to increase the risk of osteoporosis. Current guidelines advocate a baseline dual-energy x-ray absorptiometry (DEXA) and risk-stratified approach to managing bone health. Local guidelines produce a risk-stratified action plan for primary care. Methods: A prospective review of consecutive breast cancer patients attending their routine 1-year follow-up appointment allowed completion of the database. For this audit we have selected the subset of patients over the age of 65 years at diagnosis. Demographic and treatment data was collected from hospital notes, electronic records (Unity/Notis/ Ormis) or directly from the patient and prescription data from the nationally held summary care record database (SCR). Results: Data was collected from a total of 52 patients over the age of 65 at diagnosis (65-91 years). In our series 11 patients were ER negative $(21 \%)$ and four had DCIS (8\%) only. Hormone therapy was indicated for the remaining 37 patients, with two declining treatment. 32 received an AI and three tamoxifen. A total of 31/32 patients underwent a DEXA scan, which determined 11 as high risk ( $\mathrm{t}<-2$ ). Within this subgroup, six patients were further assessed for secondary causes of osteoporosis (54\%). Subsequently, $7 / 11$ patients were prescribed bisphosphonates and 8/11 vitamin D supplements (64\% and 73\%, respectively). Discussion: Our data suggest that we are assessing baseline BMD when initiating AI treatment, but risk-stratified action plans do not appear to be followed through, with only half receiving appropriate therapy to protect bone health. 


\title{
SUPPLEMENT
}

\author{
P6. Frailty assessment of older women with breast cancer in a breast unit within a district general hospital \\ Caroline Baya ${ }^{1,2}$ \\ 'Macmillan Breast CNS and Older Person's Nurse Fellow, Kings' College London, London, UK \\ 2Princess Alexandra NHS Hospital Trust, Epping Breast Screening Unit, UK
}

Background: With an increasing number of older people being diagnosed with cancer and with poorer mortality rate for patients over the age of 75 years, there is a national strategy (1) for improving the outcomes that the NHS delivers to this patient group. A need to improve a comprehensive care pathway that will include frailty screening and comprehensive geriatric assessment for older and frailer patients being diagnosed with cancer and undergoing treatment. (2) Breast cancer in the elderly is a disease associated with comorbidities and frailty, and with an increased risk of dying from comorbidities except the cancer itself. Methods: A Quality Improvement Project using the PDSA cycle: Plan - Do - Study - Act method.

- Cycle 1: Early structured holistic assessment;

- Cycle 2: Training of nursing staff relating to age-related conditions, frailty and holistic needs assessment of older people;

- Cycle 3: Test the Edmonton Frail Scale on eight patients.

The Edmonton Frail Scale (3) is a tool that is used in an outpatient surgical setting and the tool tests 10 domains relating to cognition, general health, functional independence and performance, mood, social support, medication and continence. Results: Since the start of the project all breast cancer patients (25) aged 75+ years completed structured comprehensive assessment early in the patient's pathway. The referral rates for specialities/therapies and primary care have increased by $24 \%$ (3-month period 2015 audited against 3-month period 2016). The Edmonton Frail Scale is now embedded as part of standard assessment of all women aged $75+$ years with breast cancer. Discussion: Using a frailty tool - this is a guide to identify domains of frailty that are amenable to preoperative optimisation as well as determining levels of frailty that requires a Comprehensive Geriatric Assessment. Comprehensive assessments, care planning and referral activities require nursing time. MDT treatment decision-making process - improved focus on the individual's needs and preferences.

\section{Acknowledgement}

Mr A Patel (Princess Alexandra NHS Trust) and Dr C Naughton (Kings College London).

\section{References}

1 Independent Cancer Taskforce. Achieving World-Class Cancer Outcomes. A Strategy for England 2015-2020. London, UK (2015).

2 Society of Geriatrics Oncology (2016). www.siog.org/content/comprehensive-geriatric-assessment-cga-older-patient-cancer

3 British Geriatrics Society. Fit for Frailty. Consensus best practice guidance for the care of older people living with frailty in the community and outpatient settings. BGS, London, UK (2014).

\section{P7. Breast reconstruction in elderly women}

H Byrne', N Nagrath', F Aloraifi', L Fopp', MS Irwin', A Agrawal' \& SL Benyon'

'Cambridge University Hospitals NHS Trust, Hills Road, Cambridge, CB2 0QQ, UK

Background: Breast cancer is most prevalent in the elderly age group. A major contributor to an improvement of breast cancer treatment and quality of life has been the opportunity to offer patients breast reconstruction. Evidence has shown that age is not a risk factor for poor $s$ the outcome. We would like to present a retrospective audit comparing the elderly population to those in a younger cohort. Particular emphasis has been placed on type of reconstructions, co-morbidities, outcomes and post-operative complications. Methods: We retrospectively reviewed the authors' experience with breast reconstruction between Jan. 2015 and Sept. 2016. EPIC (computerised patient record system) was used to collect data from pre-operative and post-operative consultations, as well as operating lists. Patients aged $\geq 55$ years were defined as 'elderly'. Length of hospital stay (LOS), smoking history, co-morbidities, type of reconstruction, oncological treatment and postoperative outcome were compared between the 'elderly' and 'younger' patient groups. Results: During the 21 month period, 171 women underwent breast reconstruction; $69 \%(\mathrm{n}=118)$ of these were women $<55$ years old. A greater proportion of the elderly (26\%) compared to the young (4\%) had a history of vascular disease. The mean LOS was similar between groups (5.8 [young] vs 6.1 [elderly] days). Considering reconstruction, the same proportion of women in both cohorts underwent free flaps (45\%), whilst a greater percentage of young women (42\%) underwent Implant+ADM reconstruction compared to the elderly cohort (26\%). No adverse outcomes occurred in $58 \%$ of young and $47 \%$ of elderly women. There was no statistical difference between age group and outcome $(\mathrm{p}=0.181)$. Discussion: Reconstruction after mastectomy is well tolerated in the elderly. The incidence of serious complications was low and comparable in both the young and elderly groups. Careful patient selection is key to a successful reconstruction. 
P8. Biological subtyping and response to primary endocrine therapy in older women with early operable primary breast cancer - a study based on core needle biopsy

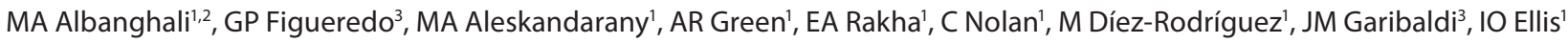

\section{\& KL Cheung}

'School of Medicine, University of Nottingham, UK

${ }^{2}$ Faculty of Applied Medical Sciences, Al Baha University, KSA

${ }^{3}$ School of Computer Science, the Advanced Data Analysis Centre, University of Nottingham, UK

Introduction: Biological subtypes of breast cancer (BC) have an essential predictive value in determining clinical behaviours of BC and responses to different treatment modalities. However, knowledge related to the biological predictors of response to primary endocrine therapy (PET) remains limited. Objective: To determine the biological subtypes of primary BC in older women and correlate them with observed responses to PET. Methods: Tissue microarray (TMA) blocks were successfully constructed using diagnostic core needle biopsies (CNB) from Nottingham series $(n=1700+)$ of older ( $\geq 70$ years) women with early operable primary BC (clinical size $\leq 5 \mathrm{~cm}$ ), followed up for more 30 years. Out of this series, 196 cases who received PET were immunohistochemically stained and scored for a panel of 17 biomarkers (ER, PgR, Ki67, p53, EGFR, HER2, HER3, HER4, BCL2, CK5/6, CK7/8, MUC1, VEGF, PTEN, AIB1, LKB1 and BRCA1). Clustering analysis was then performed utilising unsupervised K-means and partitioning around medoids algorithms. Assessment of response to PET was performed using the International Union Against Cancer criteria. Result: Three clusters were identified, including luminal A (53\%), B (28\%) and low ER luminal (19\%). When compared with luminal B and low ER luminal subtypes, luminal A subtype was associated with a higher objective response rate at 6 months (54 vs 38 and 42\%, respectively), longer time to progression (TTP; median: 96 vs 30 and 35 months, respectively; p < 0.01) and higher 5-year breast cancer-specific survival (91 vs 75 and 69\%, respectively; p < 0.01). Conclusion: The success in development of TMA from the limited CNB material allows comprehensive study of biology from nonoperatively treated BC older patients. Biological subtypes showed significant value in terms of defining a subgroup of patients (luminal A) with low risk of disease relapse and longer TTP. Further studies should consider the benefits of using biological subtypes in defining appropriate PET agents.

\section{P9. Comprehensive biology profiling of oestrogen receptor-positive breast cancer in older women - a study based on core needle biopsy}

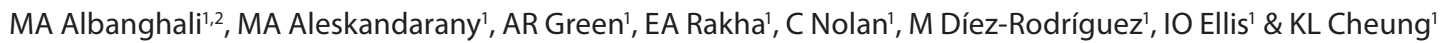

'School of Medicine, University of Nottingham, UK

2Faculty of Applied Medical Sciences, Al Baha University, KSA

Introduction: The biology of breast cancer (BC) plays an essential role in predicting clinical outcomes and response to treatment. Profiling of tumour based on their biology utilising diagnostic core needle biopsy (CNB) instead of surgical excision would encompass all patients regardless of their primary treatments. Objective: To determine the biological profile of oestrogen receptor (ER)-positive early operable (clinical size $\leq 5 \mathrm{~cm}$ ) primary breast cancer in older ( $\geq 70$ years) women, utilising a previously developed technique for constructing tissue microarray (TMA) from diagnostic CNB [1]. Methods: Of a consecutive series of 1700+ older women (with follow-up for more than 30 years in a dedicated clinic), TMA blocks were successfully constructed from diagnostic CNBs. Within this series of patients, 536 cases with ER-positive (H-score $\geq 50)$ tumour were involved in this study. Results: Immunohistochemical analysis indicated high expression $(\geq 50 \%)$ of $\mathrm{PgR}(78 \%)$, CK7/8 (99.6\%) and BRCA1 (70\%), and low expression ( $\leq 50 \%)$ of Ki67 (16\%), EGFR (2\%), HER2 (0.2\%), HER3 (33\%), HER4 (30\%), p53 (11\%), CK5/6 (12\%), MUC1 (23\%), LKB1 (31\%), BCL-2 (45\%), PTEN (15\%) and AIB1 (46\%). Furthermore, 47\% of cases were found to express ER with H-score >100, and 39\% with H-score $>200$. PgR and BCL-2 were associated with statistically significantly better breast cancer specific survival (BCSS), while Ki67, MUC1 and p53 were inversely correlated with BCSS. PgR (HR: 0.43; p = 0.015), Ki67 (HR: 3.13; p = 0.001) and MUC1 (HR: 2.051; $\mathrm{p}=0.033)$ retained their prognostic significance on multivariate analysis. Five-year BCSS rates for positive versus negative expressions of PgR, Ki67 and MUC1 were 91, 51 and 83\% versus 81, 98 and 99\%, respectively. Conclusion: ER-positive BCs in older women appear to show high expression of good prognostic markers and low expression of poor prognostic markers, with PgR, Ki67 and MUC1 having independent prognostic significance.

\section{Reference}

1 Albanghali M, Rakha E, Green A et al. Potential of a novel technique for constructing tissue microarrays from core needle biopsy as seen in older women with primary breast cancer. 2016 ASCO Annual Meeting 3-7 June 2016, Chicago, IL, USA. J. Clin. Oncol. 34 (2016) (suppl; abstr e23283). 
P10. Age standardised molecular pattern of early operable primary breast cancer

BM Syed $^{1,2}$, AR Green', IO Ellis \& KL Cheung ${ }^{1}$

'School of Medicine, University of Nottingham, UK

${ }^{2}$ Medical Research Centre, Liaquat University of Medical \& Health Sciences, Jamshoro, Pakistan

Introduction: Emerging evidence suggests that breast cancer in the older population tends to have more favourable biology. This study aimed to explore a possible differing pattern of tumour biology according to age. Methods: The study included 2383 women with T0-2N0-1M0 breast carcinoma managed in a single institution in Nottingham. Among these patients 575 were $\geq 70$ years of age derived from a consecutive series. The younger (<70 years) patients ( $\mathrm{n}=1808)$ were from a previously characterised, consecutive series. All patients were treated by primary surgery with tissue microarrays constructed from their surgical specimens. Indirect immunohistochemsitry was used for analysis of a panel of biomarkers. Results: There were age-related changes in the pattern of positivity of ER ( $p<0.001)$, PR ( $p=0.003)$, E-Cadherin $(p<0.001), K i 67(p<0.001), p 53(p<0.001)$, CK5/6 (p < 0.001), CK7/8 $(p=0.006)$, CK14 (p < 0.001), CK17 (p = 0.03), CK18 (p < 0.001) and bcl2 (p<0.001). The following patterns were observed:

A. A gradual rise starting at 40 years - ER, PgR, Muc1 and CK18;

B. A gradual decline starting at 40 years - Ki67, HER2, CK17 and E-Cadherin;

C. A rise at 70 years $-\mathrm{Bcl} 2, \mathrm{CK} 5 / 6$ and $\mathrm{CK} 14$;

D. Two peaks at 40/50 years and at 70 years - CK7/8, CK19, and p53.

Discussion: Biology of breast cancer shows a differing pattern according to age, with 40 and 70 years being key milestones. The pattern suggests $<40$ years as representing an aggressive phenotype, $>70$ years as favourable and between 40 and 70 years as the transition. Recognition of such pattern supports the use of a personalised treatment approach based on precision biological assessment. 